\title{
MARS: A Personalised Mobile Activity Recognition System
}

\author{
João Bártolo Gomes*, Shonali Krishnaswamy*, Mohamed M. Gaber ${ }^{\dagger}$, \\ Pedro A.C. Sousa ${ }^{\ddagger}$, and Ernestina Menasalvas ${ }^{\S}$ \\ * Institute for Infocomm Research (I2R), A*STAR, Singapore \\ bartologjpdi2r.a-star.edu.sg, spkrishnadi2r.a-star.edu.sg \\ ${ }^{\dagger}$ School of Computing, University of Portsmouth, United Kingdom \\ mohamed.gaber@port.ac.uk \\ ${ }^{\ddagger}$ Faculdade de Ciências e Tecnologia, Universidade Nova de Lisboa, Caparica, Portugal \\ pasefct.unl.pt \\ §acultad de Informatica, Universidad Politecnica Madrid, Spain \\ emenasalvasefi.upm.es
}

\begin{abstract}
Mobile activity recognition focuses on inferring the current activities of a mobile user by leveraging the sensory data that is available on today's smart phones. The state of the art in mobile activity recognition uses traditional classification learning techniques. Thus, the learning process typically involves: i) collection of labelled sensory data that is transferred and collated in a centralised repository; ii) model building where the classification model is trained and tested using the collected data; iii) a model deployment stage where the learnt model is deployed on-board a mobile device for identifying activities based on new sensory data. In this paper, we demonstrate the Mobile Activity Recognition System (MARS) where for the first time the model is built and continuously updated on-board the mobile device itself using data stream mining. The advantages of the on-board approach are that it allows model personalisation and increased privacy as the data is not sent to any external site. Furthermore, when the user or its activity profile changes MARS enables promptly adaptation. MARS has been implemented on the Android platform to demonstrate that it can achieve accurate mobile activity recognition. Moreover, we can show in practise that MARS quickly adapts to user profile changes while at the same time being scalable and efficient in terms of consumption of the device resources.
\end{abstract}

\section{INTRODUCTION}

Mobile activity recognition (AR) is a popular area of research in pervasive computing due to its importance for context-aware applications. These applications belong to a wide range of domains, including healthcare [11], sustainable transportation [1], and social networking [7].

The general objective for AR from sensory data is to analyse such continuous data and identify the occurrence of the activities of interest with high accuracy. In particular, mobile AR focuses on inferring the current activities of a mobile user by leveraging the rich sensory data that is available on today's smart phones.

Mobile AR is usually formulated as a classification problem, where supervised machine learning is used to interpret sensed data into activities [2], [9]. The learning process normally goes through the following stages: i) data collection, where sensor data is collected over a specified period of time from one or more mobile users, with the users typically labelling/annotating their activities; ii) data transfer, where the collected data is transferred and collated in a centralised repository; iii) learning/model building, where the AR classification model is trained and tested using the collected data; iv) model deployment, where the learnt model is deployed on-board the mobile device for identifying and classifying activities from sensory data. These state of the art mobile AR approaches from ubiquitous sensors have been shown to achieve high recognition rates [9]. This may give the impression that the general problem of AR has been solved successfully. However, in existing approaches the obtained models are static, are built off-line in an external (to the mobile device) environment and little attention is given to issues such as personalisation of the model and privacy.

To address these issues, we propose to demonstrate a prototype of the Mobile Activity Recognition System (MARS) implemented on the Android mobile platform. MARS learns the classification model on-board the mobile device itself through ubiquitous data stream mining in an incremental manner. The main advantages of on-board mobile data stream mining for mobile activity recognition are:

- dynamic instead of static model building which facilitates the adaptation of the classifier as the user's activity profile changes.

- higher personalisation as the training data is subjectspecific and its continuous nature reflects current user behaviour.

- privacy preserving and as the data is not sent to an external site.

- reduced communication overheads in terms of bandwidth use/data transfer as well as battery drain/usage since local processing is less expensive than regular data transfer.

The rest of the paper is organised as follows. The following Section reviews the related work. Section III presents the definition of mobile AR as classification problem, which is fol- 
lowed by a detailed description of the existing open challenges in Section IV. The proposed Mobile Activity Recognition System (MARS) is presented Section V. Finally, in Section VII, we describe the scenario for the demonstration.

\section{RELATED WORK}

AR from sensor data is a popular research field that has contributed with several high recognition rate approaches. Many of these use supervised machine learning algorithms, such as Decision Trees [2], Artificial Neural Networks, Hidden Markov Models, Naive Bayes, K-Nearest Neighbour or Support Vector Machines. For an extensive review of supervised learning approaches for AR please refer to [9]. Here we focus our review on works that perform mobile AR from sensor data.

One of the most cited publications on activity recognition in pervasive computing [2] deployed five small biaxial accelerometers worn simultaneously on different body positions in order to distinguish 20 activities of interest. The data was collected from 20 subjects that annotated it themselves without researcher supervision or observation. From the learning algorithms tested, $\mathrm{C} 4.5$ decision trees showed the best performance with an overall accuracy rate of $84 \%$. Such technique is considered to be slow to train but quick to run. Therefore, the authors suggest that a pre-trained decision tree should be able to recognise user activities in real-time on a 2004 top-end mobile device. Moreover, it is reported that some activities are recognised with subject-independent training data while others seem to require subject-specific training data.

In [10], a system that uses the iPhone for basic activity recognition (i.e., running, walking, bicycling, and sitting) is proposed. The system provides a set of open source tools: i) iLog: mobile tool for collecting training data; ii) iModel: Java application that uses WEKA algorithms for building off-line activity models from iLog data; ii) iClassify: deploying iModel models generated off-line on the iPhone to provide real-time activity classification to applications. The experiments using data from 8 subjects report a mean classification accuracy of 99.48\% using a within-person model and $97.4 \%$ when using a cross-person model, again implying that subject-specific data may achieve higher accuracy due to higher personalisation.

Recently, [6] proposes and experimentally evaluates a system that uses phone-based accelerometers to perform mobile AR. Data was collected from 29 subjects as they performed their daily activities such as walking, jogging, climbing stairs, sitting, and standing. This works shows how a smart phone (Android) can be used to perform activity recognition, simply by carrying it in a fixed position (front pants leg pocket). The results show that most activities are recognised correctly over $90 \%$ of the time. Still, the collected data is transferred to an Internet-based server where a static model is generated offline. Again the issues of personalisation or privacy are not addressed. Still, in the future work section it is mentioned that an improvement of the proposed system would be to generate the model on-board. Nevertheless, to the best of our knowledge such improvement has not yet been proposed.
The reviewed approaches [2], [10], [6] built static classification models off-line in an external (to the mobile device) environment. Moreover, the streaming nature of sensorial data is not taken into account nor the possibility that the model needs to be adapted over time. In addition, little attention has been given to the personalisation of the built model to suit a particular user, despite the results that seem to indicate that better accuracy is obtained with personalised models (i.e., training and test data from the same subject). To the best of our knowledge, no other ubiquitous data stream mining approach has been proposed so far to address on-board mobile AR.

For a more extensive review of data stream mining systems that have been used successfully in other applications please refer to [5].

\section{PROBLEM DEFINITION}

Let $X$ be the space of features generated from the available input sensors and $Y$ be the set of possible (discrete) class labels that correspond to the activities of interest. Consider a data stream $D S$, where $X_{i}=\left(\overrightarrow{x_{i}}, y_{i}\right)$ with $x_{i} \in X$ and $y_{i} \in Y$, represents the $i^{t h}$ record in DS. The modelling of AR is formulated as a function $f$ that assigns each sensor feature input record $\overrightarrow{x_{i}}$ to the true activity label $y_{i}$. This function $f$ can be approximated using supervised learning by training a model $m$. The goal is that the trained model $m$ minimises the number of wrongly recognised activities (i.e., achieves high accuracy).

Also it is important to note that the user activity profile can change (i.e., change in the distribution $\mathrm{P}(\mathrm{X}, \mathrm{Y})$ ) and that the model $m$ needs to be updated to reflect the most recent user activity profile.

\section{OPEN CHALLENGES}

Despite the good results of existing supervised learning approaches in AR, there are still open challenges that to the best of our knowledge have not been addressed. The following subsections introduce such challenges.

\section{A. Personal training data}

The usual supervised learning approach to AR assumes that there is abundant training data and that the function $f$ to model is static. However, in realistic situations, $f$ is usually subject dependent and can even change over time within subject. Moreover, past work shows that if the training data is collected from the subject of interest then there is no advantage to use additional training data from other subjects [8]. Still, in the case where training data from the subject of interest is not available, having data from higher number of subjects is beneficial to the resulting recognition accuracy. Moreover, the reviewed existing work in Section II also indicates that, whenever possible, the training data should be collected in a subject dependent way, while performing the activities of interest in a real-world scenario. 


\section{B. Model generation}

In most existing supervised learning approaches to mobile $\mathrm{AR}$, the training data is collected, a classification model is generated offline from the collected data, and finally the obtained model is deployed. Nevertheless, there are disadvantages that can result from using this type of offline learning process:

- The obtained model is static - Once a model is generated it does not incorporate new information.

- Computational costs - The batch algorithms typically used to generate the model are not designed to be executed in mobile devices. Such algorithms usually require several passes over the dataset and require that the entire dataset is allocated into main memory. In contrast, ubiquitous data stream mining approaches process each record only once and are memory efficient [3], [5].

\section{MARS: MobiLe ACTIVITy RECOGNITION System}

MARS is a ubiquitous data stream mining approach to mobile AR. Such approach is motivated and focused on addressing the open challenges described in the previous section. Conversely to traditional supervised learning, data stream classification algorithms are able to update an anytime model $m_{t}$ (i.e., model at time $t$ ) as new training records are available in the stream. Moreover, these algorithms are lightweight and can be executed using the computational resources usually available on nowadays mobile devices. MARS enables greater personalisation and privacy while bringing the whole learning process on-board the mobile device.

\section{A. Learning the model}

During the training phase the user performs the activities of interest and annotates interactively the data collected from the sensors using a user-friendly interface (i.e., usually simply by selecting from a list the activity that he previously executed). This type of naturalistic data collection has been successfully used before, however, the records are saved to be then processed by a offline learning algorithm, while we propose that the annotated data stream should be processed on-board by an incremental learning algorithm. Another option is that when MARS is deployed, a pre-trained model(s) is used and the user simply corrects model $m_{t}$ in its predictions. Figure 1 illustrates the MARS training process.

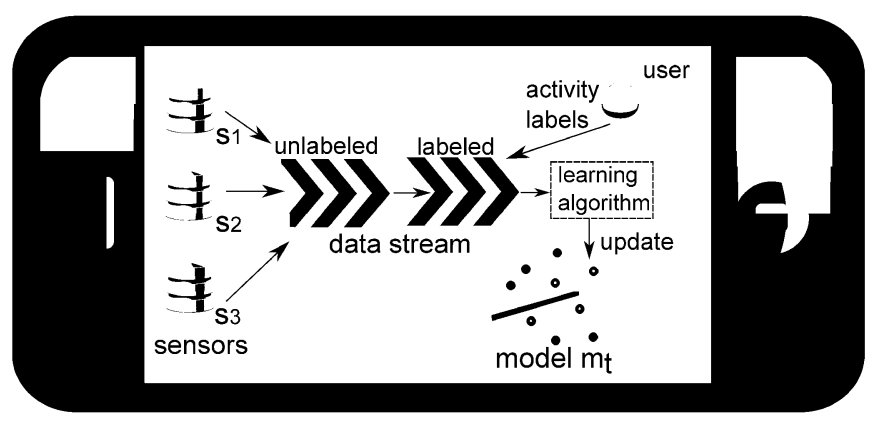

Fig. 1. MARS: Training process of anytime model $m_{t}$
To classify new records (unlabelled) the anytime model $m_{t}$ is used to simply return the predicted activity.

Since an anytime model $m_{t}$ is assumed, it is possible to keep an estimate of the $m_{t}$ accuracy as new training records are incorporated. For this purpose we propose that the prequential statistic is used [4].

In real world situations is important to be able to adapt the anytime model. For instance, adapting the model to a new living environments or new subject behaviour. In such situations it is likely that the most recent training records represent the activities of interest and less importance should be given to older records that represent past behaviour.

The code for the MARS process can be found in Algorithm V-A.

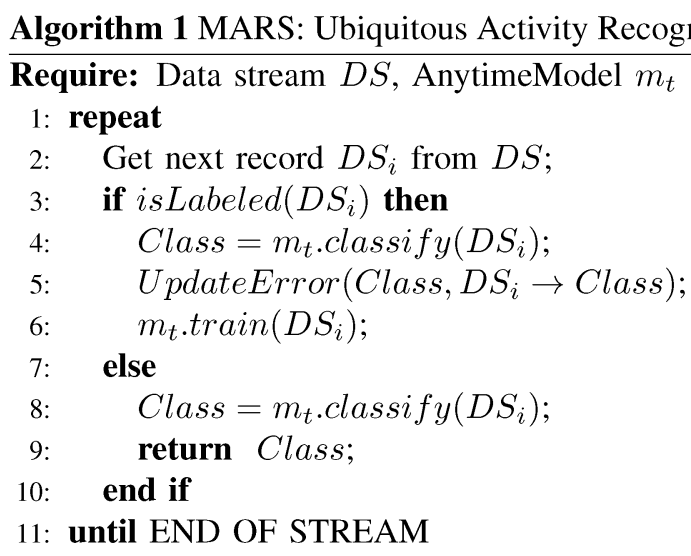

\section{IMPLEMENTATION OF THE MARS PROTOTYPE}

We implemented a prototype of MARS on the Android platform. The prototype for this demo has been deployed and tested on a low-end Android phone, ZTE Blade, sold in UK as Orange San Francisco, that in early 2011 was one of the budget Android phones on the market. The phone has a Qualcomm MSM7227 $600 \mathrm{MHz}$ processor, 512MB of RAM, $1250 \mathrm{mAh}$ battery and runs Android 2.2 Froyo.

The Naive Bayes classifier was used as learning algorithm in our prototype. We selected this algorithm because it provides a simple, incremental yet efficient approach to learn probabilistic knowledge. The implementation of the algorithm that is available in the MOA framework [3] is used. Since the MOA implementation is developed in Java it was easy to deploy it on the Android prototype where it executes efficiently without any problems.

Some changes have been made, so the learning algorithm is more accurate and efficient for mobile activity recognition. These changes are discussed in the following subsections.

\section{A. Adapting the model}

The simplest solution to achieve model adaptation is that the anytime model represents only the most recent records that belong to a sliding window of fixed size. However, in general computing statistics over sliding windows requires storing all the records inside the window into memory. A solution that 
can be used alternatively or when it is not possible to store all the records within the window in memory, is to weighting the records accordingly to their age.

For the Naive Bayes algorithm used in the prototype, we used the weighting solution that in our particular case consists in multiplying the stored statistics of the Naive Bayes algorithm by $\alpha$ fading factor a $(0<\alpha<1)$ without any additional memory cost, and then update the model with the new information. A higher $\alpha$ means more gradual forgetting while a lower value indicates that a faster adaption.

\section{B. Sensors and Features}

For this implementation, we extracted features only from the accelerometer sensor as it is the most commonly available sensor in commodity mobile phones. Nevertheless, the current implementation can also easily integrate more sensors available in the Android platform or wearable sensors that can be accessed through bluetooth.

For the accelerometer sensor the Euclidean norm is calculated for each readings. Moreover, a sliding window of these norms is considered. From this window the features $X$ used are the minimum and maximum value within the window.

\section{Basic application}

The implementation can be used as a service but also provides a simple application that monitors the time the user spends on each of its activities. This can be useful for a user that wants to monitor if he performs at least 3 hours of physical activity a day.

\section{DEMO SCENARIO}

The scenario proposed is highly interactive. The users are given mobile phones and are expected to play with them to evaluate how MARS can accurately recognise their activity. Moreover, the users are encouraged to train the device to recognise their personal activity profile. For instance, a user may find that what MARS is recognising as running is more like jogging to himself. The user can update the model in real time, providing MARS an example of what the running activity should be like.

Figure 2 shows the interface of the MARS prototype, on the left we can see the training interface where a user selects the activity being performed and starts/stops the training process. A graph showing the sensor readings is displayed to provide a more interactive training environment. On the right we can see the interface that displays the activity being performed. In here we see a pie chart where each colour represents an activity and the model confidence in that prediction. Therefore, the user will typically see the colour of this pie changing with his activity.

For the demo we plan to consider simply a simple set of activities as can be $Y=\{W$ alking, Running, Still $\}$. If time and the local constraints allow, we would like to demonstrate how easy it is to further tune the prototype, changing, the set of activities, the sensors and features used. This will demonstrate how MARS can be used as framework for future research in

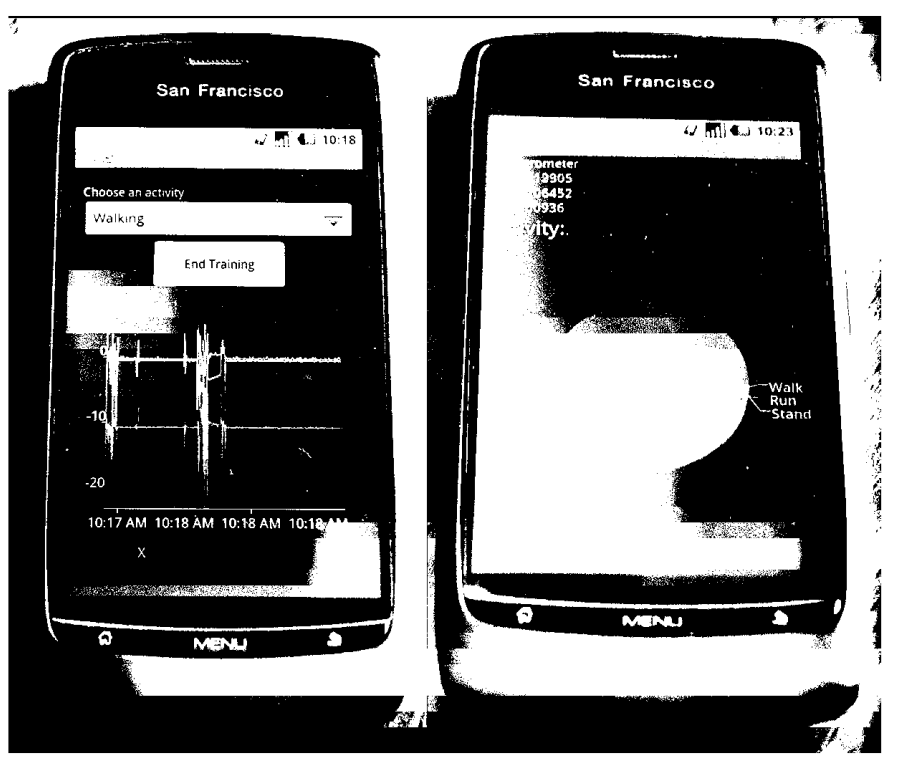

Fig. 2. MARS prototype interface

AR. Therefore, encouraging the collaboration and exchange of ideas with researchers and experts in the field and provide valuable feedback for future versions of our system.

\section{REFERENCES}

[1] E. Agapie, G. Chen, D. Houston, E. Howard, J. Kim, MY Mun, A. Mondschein, S. Reddy, R. Rosario, J. Ryder, et al. Seeing our signals: Combining location traces and web-based models for personal discovery. In Proceedings of the 9th workshop on Mobile computing systems and applications, pages 6-10. ACM, 2008.

[2] L. Bao and S.S. Intille. Activity recognition from user-annotated acceleration data. Pervasive Computing, pages 1-17, 2004.

[3] A. Bifet, G. Holmes, R. Kirkby, and B. Pfahringer. Moa: Massive online analysis. The Journal of Machine Learning Research, 11:1601-1604, 2010.

[4] J. Gama, R. Sebastiao, and P.P. Rodrigues. Issues in evaluation of stream learning algorithms. In Proceedings of the 15th ACM SIGKDD international conference on Knowledge discovery and data mining, pages 329-338. ACM New York, NY, USA, 2009.

[5] S. Krishnaswamy, J. Gama, and M.M. Gaber. Advances in data stream mining for mobile and ubiquitous environments. In Proceedings of the 20th ACM international conference on Information and knowledge management, pages 2607-2608. ACM, 2011.

[6] J.R. Kwapisz, G.M. Weiss, and S.A. Moore. Activity recognition using cell phone accelerometers. ACM SIGKDD Explorations Newsletter, 12(2):74-82, 2011

[7] E. Miluzzo, N. Lane, S. Eisenman, and A. Campbell. Cenceme-injecting sensing presence into social networking applications. Smart Sensing and Context, pages 1-28, 2007.

[8] N. Ogawa, K. Kaji, and N. Kawaguchi. Effects of number of subjects on activity recognition-findings from hasc2010corpus. In International Workshop on Frontiers in Activity Recognition using Pervasive Sensing (IWFAR2011), pages 48-51, 2011.

[9] S Preece, Jy Goulermas, Lpj Kenney, D Howard, K Meijer, and $\mathrm{R}$ Crompton. Activity identification using body-mounted sensors a review of classification techniques. Physiological Measurement, 30(4):R1R33, 2009.

[10] T. Saponas, J. Lester, J. Froehlich, J. Fogarty, and J. Landay. ilearn on the iphone: Real-time human activity classification on commodity mobile phones. University of Washington CSE Tech Report UW-CSE08-04-02, 2008.

[11] J. Yang. Toward physical activity diary: Motion recognition using simple acceleration features with mobile phones. In Proceedings of the 1st international workshop on Interactive multimedia for consumer electronics, pages 1-10. ACM, 2009. 